\section{NEW MENTAL HEALTH WELLNESS INITIATIVE LAUNCHED}

A new dental initiative has been launched to encourage all dental workplaces to make Mental Health Wellness a priority.

The Mental Health Wellness strategic steering group was formed through the Dental Professional Alliance, specifically to co-design, develop and maintain a framework that encourages and enables all dental professionals to act in a timely, appropriate, and safe manner when identifying Mental Health Wellness issues in the workplace. The strategic steering group has been led and Chaired by Fiona Ellwood BEM DPA, a trained and practising Mental Health First Aider, and Roz McMullan, Chair of Probing Stress in Dentistry in Northern Ireland.

The initiative has gained widespread support from across dentistry, with a large number of endorsing partners.

Mental health is never far from front page news and it has a longstanding association with dentistry. When you look closer this appears to be associated with dentists rather than the whole dental team. To anyone that understands the intricacies of being part of a dental team and especially the clinical team, it is difficult to perhaps see why there have only ever been papers and research on dentists.
Yes, there are added pressures, but if pressures spill over it can very quickly become a team matter. Support for the whole team is varied across settings and across team members, yet mental health knows no such boundaries.

There have been several papers produced during the pandemic about stress, anxiety, and burnout; most have again focused on the dentist. When you look to the NHS in general, it talks more broadly of frontline staff, although much has been written about the nursing teams too. While we know that stress, anxiety and burnout are not mental illnesses we know that they can add to mental health issues. In an almost timely fashion, the Office for National Statistics ${ }^{1}$ has just published data on Coronavirus and Depression in Adults and this paints a worrying picture. Double the number of adults have experienced depression symptoms in comparison to the pre-COVID statistics. ${ }^{1}$ In Jan-March 2021 1-5 (21\%) have suffered with depression symptoms, with an increase from Nov 2020 which was $19 \% .{ }^{1}$ Notably, the evidence shows that 4-10 cases are young adults and female. ${ }^{1}$

Humphris et al. ${ }^{2}$ have just published a paper looking at health and wellbeing as an outcome of uncertainties of the pandemic in dental team

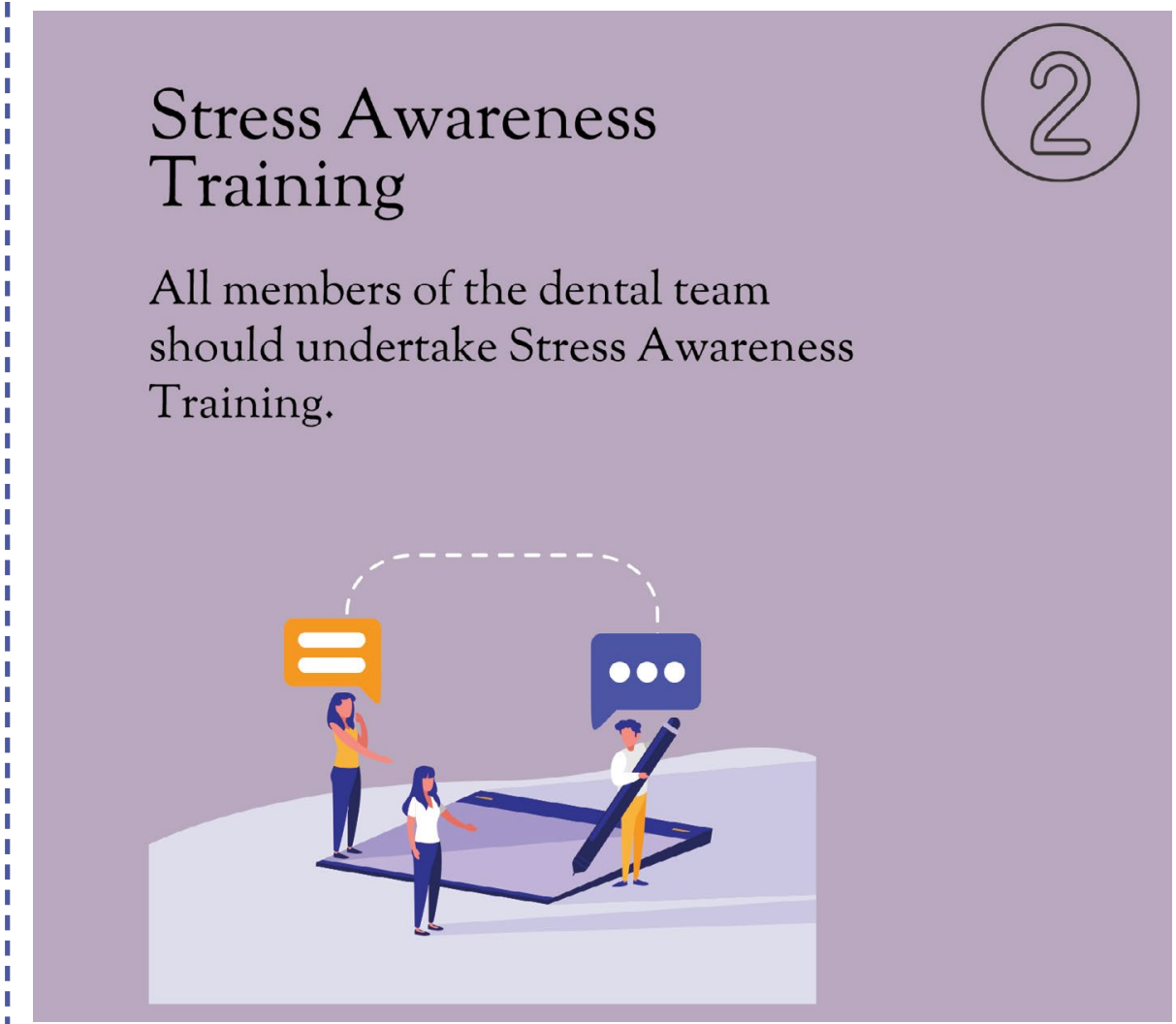

members. Interestingly the paper suggests that primary care staff reported higher incidences. What is of great interest is the recommendations which clearly suggest that there could be benefit in 'resourcing staff support and interventions to help team members in [challenging times]. ${ }^{2}$

Not only does the Humphris et al. ${ }^{2}$ paper speak to the whole team it touches on the narrative of resourcing staff support and interventions, which takes this full circle and right back to the principles of the extensive work recently undertaken by the Dental Professional Alliance and stakeholders, who have designed a framework introducing the need for a mental health wellness lead in every dental setting with an underlying ethos that early intervention and safe signposting is paramount.

The initial call to action from the initiative is that each dental workplace should have an identified individual who acts as a 'Mental Health Wellness Lead' and who through a recognised training programme is confident, competent and committed to improving the perception of mental health wellness in the workplace. This forms part of a six-part process:

- Identify Mental Health Wellness Lead

- All members of dental team to undergo Stress Awareness Training

- Leads to undertake MHFA training

- Design workplace action plan

- Join local peer support networks

- Complete annual training and maintain skills.

For more information about the initiative and how you can get involved, visit: https:// mhwd.org/.

\section{References}

1. Office for National Statistics. Coronavirus and depression in adults, Great Britain: January to March 2021. Analysis of the proportion of the British adult population experiencing some form of depression in early 2021 by age, sex and other characteristics. 5 May 2021. Available at: https://bit.ly/2QqfHoo (accessed May 2021).

2. Humphris G, Knights J, Beaton L et al. Exploring the effect of the COVID19 pandemic on the dental team: preparedness, psychological impacts and emotional reactions. Front Oral Health 2021 https://doi.org/10.3389/ froh.2021.669752. 